\title{
An overview of the reliability prediction related aspects of high power IGBTs in wind power applications
}

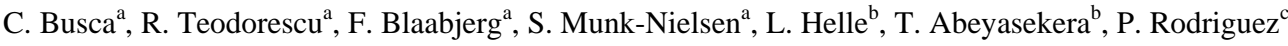 \\ a Department of Energy Technology, Aalborg University, Aalborg, Pontoppidanstraede 101, 9220, Denmark \\ ${ }^{\mathrm{b}}$ Vestas Wind System A/S, Aarhus, Hedeager 42, 9220, Denmark \\ ${ }^{c}$ Department of Electrical Engineering, Technical University of Catalonia, Terrassa, 08222, C. Colom 1, Spain
}

\begin{abstract}
Reliability is becoming more and more important as the size and number of installed Wind Turbines (WTs) increases. Very high reliability is especially important for offshore WTs because the maintenance and repair of such WTs in case of failures can be very expensive. WT manufacturers need to consider the reliability aspect when they design new power converters. By designing the power converter considering the reliability aspect the manufacturer can guarantee that the end product will ensure high availability. This paper represents an overview of the various aspects of reliability prediction of high power Insulated Gate Bipolar Transistors (IGBTs) in the context of wind power applications. At first the latest developments and future predictions about wind energy are briefly discussed. Next the dominant failure mechanisms of high power IGBTs are described and the most commonly used lifetime prediction models are reviewed. Also the concept of Accelerated Life Testing (ALT) is briefly reviewed.
\end{abstract}

Keywords: IGBT, reliability, press-pack, power cycling, mission profile

\section{Introduction}

Nowadays wind energy is seeing a rapid development all around the world [1]. WTs have the advantage of not producing greenhouse gas emissions during their operation and a WT produces in about three to six months the amount energy that is needed to manufacture, operate and recycle the turbine after its useful life [2].

At the end of 2008 the cumulative wind power capacity installed in the European Union (EU) was 64.9 GW from which 63.4 GW is onshore and 1.5 GW is offshore [1]. According to the 2009 Renewable Energy Directive, by 2020 the EU should meet $20 \%$ of its energy needs using renewable sources. Wind power would account for about $230 \mathrm{GW}$ from which $190 \mathrm{GW}$ is installed onshore and $40 \mathrm{GW}$ is installed offshore [2]. There is a large predicted growth in both the onshore and offshore installed wind power capacity.

Besides the installed wind power capacity also the size of the WTs has been continuously growing. The WT technology has developed a lot during the past two and half decades [2]. The increase in terms of maximum rotor diameter is from $15 \mathrm{~m}$ (in 1985) to $126 \mathrm{~m}$ (in 2009) which means an increase by a factor of more than 8 . In terms of WT maximum rated power the increase is from $50 \mathrm{~kW}$ (in 1985) to $7 \mathrm{MW}$ (in 2009) which means an increase by a factor of 140.

The technical availability achieved by modern onshore WTs is about $95 \%$ to $99 \%$ [3]. Of course this high technical availability is achieved not only because of good reliability alone but also because of good maintenance [4]. The WT industry aims to achieve higher reliability with lower maintenance [5]. Very high reliability is especially important for offshore WTs because it can be very expensive and time consuming to maintain and repair these turbines in case of failures. WT manufacturers need to consider the reliability aspect when they design new power converters for their turbines. By designing the power converter with a certain reliability in mind they can ensure that the power converters will last long enough.

\section{Failure mechanisms of high power IGBTs}

IGBTs are power switching devices which combine the advantages of the Bipolar Junction Transistor (BJT) and Metal Oxide Semiconductor Field Effect Transistor (MOSFET). The BJTs have superior current density while the MOSFETs can be controlled by voltage [6]. Therefore IGBTs combine these two advantages of having high current density and being voltage driven.

The internal structure of a standard wire-bonded IGBT module is made up of several layers of different materials. The Silicon ( $\mathrm{Si}$ ) chip is soldered on a Direct Cooper Bonded (DCB) ceramic substrate. The DCB substrate has the double role of electrically insulating the Si chip from the base plate and conducting the heat dissipated by the chip to the cooling system. The top side of the Si chip is contacted by means of using Aluminium (Al) bond wires. Thermal interface material is applied between the base plate and heat sink in order to minimize the thermal resistance [7]. The main reliability issues of standard wire-bonded IGBT modules comes from the fact that its packaging is made up of several layers of different materials with different thermo-mechanical properties. The various materials used in the packaging have different Coefficients of Thermal Expansion (CTEs) as it is shown in Tab. 1. Therefore the difference in the CTEs combined with temperature variation leads to stress formation in the packaging.

\begin{tabular}{|l|l|}
\hline \multicolumn{2}{|l|}{ Coefficient of Thermal Expansion } \\
\hline $\mathrm{Si}$ & $2.6^{*} 10^{-6} \mathrm{~K}^{-1}$ \\
\hline $\mathrm{Cu}$ & $17.5^{*} 10^{-6} \mathrm{~K}^{-1}$ \\
\hline $\mathrm{Al}$ & $23.5^{*} 10^{-6} \mathrm{~K}^{-1}$ \\
\hline
\end{tabular}




\begin{tabular}{|l|l|}
\hline $\mathrm{AlN}$ & $4.7 * 10^{-6} \mathrm{~K}^{-1}$ \\
\hline $\mathrm{Al}_{2} \mathrm{O}_{3}$ & $6.8^{*} 10^{-6} \mathrm{~K}^{-1}$ \\
\hline $\mathrm{Si}_{3} \mathrm{~N}_{4}$ & $2.7 * 10^{-6} \mathrm{~K}^{-1}$ \\
\hline $\mathrm{Mo}$ & $5.1^{*} 10^{-6} \mathrm{~K}^{-1}$ \\
\hline
\end{tabular}

Tab. 1. The coefficient of thermal expansion for different packaging materials [6].

A comparative overview of the failure mechanisms of standard wire-bonded and press-pack IGBT modules is shown in Tab. 2. As it may be seen in Tab. 2, one failure mechanism which is common to both wire-bonded and press-pack IGBTs is the cosmic ray induced burnout. Each packaging technology has its own characteristic failure mechanisms.

\begin{tabular}{|l|l|l|}
\hline No. & Wire-bonded IGBT modules & Press-pack IGBT modules \\
\hline 1 & Bond wire lift-off & Fretting damage \\
\hline 2 & Solder joint fatigue & Spring fatigue \\
\hline 3 & Bond wire heel cracking & Spring stress relaxation \\
\hline 4 & Aluminium reconstruction & Cosmic ray induced burnout \\
\hline 5 & Cosmic ray induced burnout & \\
\hline
\end{tabular}

Tab. 2. Comparative overview of wire-bonded and press-pack IGBT failure mechanisms.

Three well known weak points inside a standard wire-bonded IGBT module are the Al bond wire - Si chip interconnection, the Si chip - DCB solder joint and the DCB - base plate solder joint [7]. One of the most common failure mechanisms of standard wire-bonded IGBT modules is the bond wire lift-off. The reason behind this failure mechanism is the combination of the CTE mismatch (between $\mathrm{Al}$ and $\mathrm{Si}$ ) with temperature variation. According to [8], the bond wire liftoff failure mechanism can be delayed by different technological countermeasures like the mounting of a strain buffer on the top of the Si chips or the use of a coating layer which glues the bond wires to the Si chip.

Another dominant failure mechanism of the standard wire-bonded IGBT modules is the solder joint fatigue. In a standard IGBT module there are two solder joints: the Si chip - DCB solder joint and the DCB - base plate solder joint. The solder joint between the DCB substrate and the base plate is the critical one because the CTE mismatch between the DCB substrate and base plate is higher than the CTE mismatch between the DCB substrate and Si [8]. As the solder joint starts to crack its heat conducting ability diminishes thus leading to an acceleration of the aging process due to the higher temperatures (positive feedback).

Bond wire heel cracking is another failure mechanism which can occur in standard wire-bonded IGBT modules. This failure mechanism can be observed usually after long power cycling tests and especially in the cases where the ultrasonic bonding process was not optimized. Bond wire heel cracking is a slower failure mechanism than bond wire lift-off and usually it does not occur in modern IGBT modules [8].

Aluminium reconstruction is a degradation mechanism which can be observed after thermal cycling. The reconstruction of the metallization can be understood as the extrusion of Al grains from the surface of the metallization (the surface becomes rough). The effect of this degradation mechanism is the reduction of the effective cross section of the metallization which leads to an increased electrical resistance [8].

Some less common failure mechanisms of the wire-bonded IGBT modules like the cracking of the Si chip, the cracking of the DCB substrate and stress corrosion of the bond wires are described in detail in [8].

A packaging technology which eliminates the dominant failure mechanisms of standard wire-bonded IGBT modules is the press-pack technology. The press-pack technology has been widely used for manufacturing of various types of high power devices like Injection Enhanced Gate Transistors (IEGTs), Integrated Gate Commutated Thyristors (IGCTs), GTOs, diodes, etc. By eliminating the bond wire lift-off and solder joint fatigue failure mechanisms, the reliability of the device is greatly improved. Another advantage of press-pack IGBTs is that they allow dual sided cooling which leads to an increased power density when compared to wire-bonded IGBT modules [9]. Nowadays a wide range of press-pack IGBTs are available from several manufacturers like Westcode [10], Toshiba [11], Poseico [12], etc.

The round type press-pack IGBTs contain no bond wires or solder joints at all. The contact to the gate, collector and emitter is made through pressure contact alone [13]. Similarly to wire-bonded IGBT modules, the press-pack IGBT contains several IGBT chips connected in parallel in order to obtain the desired current rating. In press-pack IGBTs, molybdenum plates are placed on each side of the Si chips in order to ensure a proper electrical contact and to minimize the pressure distribution non-uniformity [14]. The gate of each individual chip is connected to the external gate terminal by means of using a spring loaded contact pin and a planar distribution system. The use of a planar distribution system ensures a very low inductance path to the gate contacts of each chip. Moreover the gate resistors are embedded in the IGBT chips [15].

A special feature of the press-pack IGBT is the Short Circuit Failure Mode (SCFM). When a press-pack IGBT fails, the semiconductor within the device undergoes a metallurgical change which leads to the formation of a permanent short circuit path through the device [9]. This special property of press-pack IGBT modules offers redundancy in the case of multiple series connected devices, which is especially useful in High Voltage Direct Current (HVDC) applications.

Press-pack IGBTs eliminate the dominant failure mechanisms of standard wire-bonded IGBTs but introduce also new failure mechanisms characteristic to press-pack IGBTs like fretting damage, spring fatigue, spring stress relaxation, etc. One of the main driving forces behind the degradation of the press-pack IGBT is the same as for the standard wire-bonded IGBT modules, the CTE mismatch between the different layers of materials which are used to build the device. When the presspack IGBT is subjected to temperature variations, its internal subassemblies will expand/contract differently leading to reciprocating sliding between the subassemblies (fretting damage). Fretting damage can lead to the deterioration of the 
electrical and thermal properties of the subassemblies. The deterioration caused by fretting may be observed as an increased junction temperature [16].

Some of the new failure mechanisms characteristic to press-pack IGBTs is related to the gate contact spring and they are the spring fatigue and spring stress relaxation. Spring fatigue can occur due to the fact that during power cycling the spring may experience a very large number of compression/expansion cycles which with time can lead to the failure of the spring. Spring stress relaxation is another failure mechanism and with time it can lead to an increased contact resistance. The stress relaxation failure mechanism is material, time and temperature dependent [17].

A failure mechanism which can occur both in wire-bonded and press-pack IGBTs is the cosmic ray induced burnout. This failure mechanism doesn't has any precursors and IGBTs are especially sensitive to cosmic radiation in comparison to other devices like diodes, thyristors, GTOs, etc [8]. According to [18] primary cosmic rays are high-energy particles that are found in the space and originate from supernovae. These primary cosmic rays usually do not reach the surface of the earth but break down into numerous other high-energy particles like pions, muons, neutrons, etc. when colliding with other atmospheric particles. Cosmic rays usually cause a breakdown in the bulk of the Si chip at a random location [19]. The cosmic ray induced burnout failure mechanism is taken into account by means of the average failure rate which depends on the IGBT module type, voltage level, altitude, temperature, etc.

\section{High power IGBT lifetime prediction}

Lifetime prediction of high power IGBTs can be understood as estimating the life expectancy of the devices under certain operating conditions (stresses). For the IGBTs the stresses can be temperature, voltage, current, vibration, humidity, cosmic radiation level, etc. [20]. Lifetime prediction models of high power IGBTs are required in order to design the new WT power converters for certain reliability.

A comparative overview of the analytical lifetime models reviewed in this paper is shown in Tab. 3. The models are compared by looking at the number of model parameters and variables considered in the model. Among the studied analytical lifetime models the Bayerer model has the highest number of model parameters and variables.

\begin{tabular}{|c|c|c|c|}
\hline Equation & Lifetime model & Model parameters & Variables considered \\
\hline (1) & Coffin Manson & $a, n$ & $\Delta T$ \\
\hline (2) & Coffin Manson-Arrhenius & $a, n, E_{a}$ & $\Delta T_{j}, T_{m}$ \\
\hline (3) & Norris-Landzberg & $A, n_{1}, n_{2}, E_{a}$ & $\Delta T_{i}, T_{m}, f$ \\
\hline (4) & Bayerer & $K, \boldsymbol{\beta}_{1}, \boldsymbol{\beta}_{2}, \boldsymbol{\beta}_{3}, \boldsymbol{\beta}_{4}, \boldsymbol{\beta}_{5}, \boldsymbol{\beta}_{6}$ & $\Delta T_{i}, T_{i-\max }, t_{o n}, I, V, D$ \\
\hline (5) & Large solder joint & $L, \Delta \alpha, c, x, \gamma$ & $\Delta T$ \\
\hline
\end{tabular}

Tab. 3. Comparative overview of the analytical lifetime models for IGBTs.

According to [7] the IGBT lifetime prediction models can be split into analytical and physical models. Analytical lifetime models estimate the life of the device in terms of number of cycles to failure $N_{f}$ considering various factors such as temperature swing, medium temperature, frequency, bond wire current, etc. The main problem with analytical lifetime models is that it is difficult to accurately extract the number and amplitude of the temperature cycles from a given temperature profile (mission profile) [21]. A commonly used algorithm for the extraction of the number and amplitude of temperature cycles from a temperature profile is the rain-flow counting algorithm. Other approaches which can be used to extract the number and amplitude of thermal cycles from a temperature profile are the polynomial approach or the evaluation of the local maxima and minima [22]. There are also different thermal cycle definitions proposed in [22] which can be used to extract the number and amplitude of thermal cycles from a temperature profile. The analytical lifetime modelling of IGBTs is achieved by means of using Miner's rule for damage accumulation [7].

A simple lifetime prediction model which can be used as long as the peak of the temperature cycles does not exceed 120 ${ }^{\circ} \mathrm{C}$ is the simple Coffin-Manson model which takes into consideration only the temperatures swing $\boldsymbol{\Delta T}$ [8]. The mathematical formulation of the simple Coffin-Manson model is given in (1). The parameters $\boldsymbol{a}$ and $\boldsymbol{n}$ can be obtained by numerical simulations or experimental measurements (power cycling).

$\mathrm{N}_{\mathrm{f}}=\mathrm{a} *(\Delta \mathrm{T})^{-\mathrm{n}}$

An improved analytical lifetime model which was derived from the simple Coffin-Manson relation is shown in (2). This improved model besides the temperature swing $\boldsymbol{\Delta} \boldsymbol{T}_{j}$ takes into consideration also the medium temperature $\boldsymbol{T}_{\boldsymbol{m}}$. The medium temperature is taken into consideration by means of using an Arrhenius term. In (2) $\boldsymbol{k}$ is the Boltzmann constant and $\boldsymbol{E}_{\boldsymbol{a}}$ is the activation energy parameter which can be determined experimentally [23].

$\mathrm{N}_{\mathrm{f}}=\mathrm{a} *\left(\Delta \mathrm{T}_{\mathrm{j}}\right)^{-\mathrm{n}} * \mathrm{e}^{\mathrm{E} /(\mathrm{k} * \mathrm{Tm})}$

The Norris-Landzberg model is derived from the improved Coffin-Manson model given in (2) and it additionally takes into consideration the frequency parameter $\boldsymbol{f}$ of the temperature cycles [7]. The mathematical formulation of the NorrisLandzberg model is given in (3).

$\mathrm{N}_{\mathrm{f}}=\mathrm{A} * \mathrm{f}^{\mathrm{n} 2} *\left(\Delta \mathrm{T}_{\mathrm{j}}\right)^{-\mathrm{n} 1} * \mathrm{e}^{\mathrm{Ea} /(\mathrm{k} * \mathrm{Tm})}$ 
Another analytical model derived from the Coffin-Manson relation is the Bayerer model which is given in (4). The Bayerer model has a large number of parameters and it considers the influence of various parameters of power cycling tests and power module characteristics. In (4) $\boldsymbol{T}_{j-m a x}$ is the maximum junction temperature, $\boldsymbol{t}_{\boldsymbol{o n}}$ is the heating time, $\boldsymbol{I}$ is the applied DC current, $\boldsymbol{D}$ is the diameter of the bond wire and $\boldsymbol{V}$ is the blocking voltage. The constants $\boldsymbol{K}$ and $\boldsymbol{\beta}$ are obtained through reliability experiments [24].

$\mathrm{N}_{\mathrm{f}}=\mathrm{K} *\left(\Delta \mathrm{T}_{\mathrm{j}}\right)^{\beta 1} * \mathrm{e}^{\beta 2 /(\mathrm{Tj}-\mathrm{max}+273 \mathrm{~K})} * \mathrm{t}_{\mathrm{on}}^{\beta 3} * \mathrm{I}^{\beta 4} * \mathrm{~V}^{\beta 5} * \mathrm{D}^{\beta 6}$

Another analytical model based on the Coffin-Manson law which can be used to predict the number of cycles to failure of large solder joints is given in (5). The parameter $\boldsymbol{L}$ represents the typical lateral size of the solder joint, $\boldsymbol{\Delta} \boldsymbol{\alpha}$ is the CTE mismatch between the two surfaces which surround the solder joint, $\boldsymbol{c}$ is the fatigue exponent, $\boldsymbol{x}$ is the thickness of the solder layer and $\gamma$ is the ductility factor of the solder [8].

$\mathrm{N}_{\mathrm{f}}=0.5 *[(\mathrm{~L} * \Delta \alpha * \Delta \mathrm{T}) /(\gamma * \mathrm{x})]^{1 / \mathrm{c}}$

According to [7] in the case of physical lifetime models the knowledge of the number and amplitude of thermal cycles are not required but the failure mechanisms need to be known. Physical lifetime models are based on the knowledge of the stress-strain deformations within the device. According to [7] the physical models for the estimation of the solder joint lifetime can be split into energy, damage, stress and strain based methods. Energy based methods tend to give better results than other models because they capture test conditions with more accuracy. In the energy based models it is assumed that a device fails once the deformation energy accumulated in the device reaches a critical value. One energy based physical model is described in [7] and two other models are described in [25].

A mathematical model which can be used to predict the remaining stress in the gate spring of a press-pack IGBT is described in [26]. The mathematical formulation of the model is given in (6) where $\sigma$ is the amount of stress remaining in the material after $\boldsymbol{t}$ hours and $\boldsymbol{\sigma}_{\boldsymbol{0}}$ is the initial stress value. The constants $\boldsymbol{A}$ and $\boldsymbol{B}$ are dependent on the material and temperature.

$100 *\left(\sigma / \sigma_{0}\right)=\mathrm{A}-\mathrm{B}[\ln (\mathrm{t})]^{2}$

Another mathematical model which can be used to estimate the number of cycles to failure of gate springs in press-pack IGBTs under power cycling conditions is described in [17].

High power IGBT modules have a relatively long life time under normal use conditions therefore ALT is used for lifetime model parameterization [27]. ALT can be understood as a test method which makes a device fail faster than it would fail under normal use level conditions [28]. ALT on IGBT devices is performed with the help of thermal or power cycling setups [29].

According to [28] Accelerated life tests can be divided into qualitative and quantitative tests. Qualitative tests yield only failure modes and are also referred to as Elephant test, torture tests, highly accelerated life test, highly accelerated stress test, shake and bake tests. Qualitative accelerated life tests are generally used to see where the weak links in a device are so manufacturers will know what to improve. The results of qualitative accelerated life tests cannot be used for reliability metrics calculations. Quantitative tests are designed to quantify the life characteristics of a product under normal use conditions and therefore provide reliability information. Once the results of quantitative accelerated tests are obtained different reliability metrics like the Probability Density Function (pdf), Cumulative Distribution Function (cdf), Mean Time Between Failures (MTBF), Bx-life (the time by which x percent of the population has failed), failure rate, etc. can be calculated.

The lifetime of high power IGBTs used in WT applications can be predicted as shown in the flow diagram from Fig. 1. The input is the mission profile of the WT which can be given in terms of wind speed over time, wind speed distribution, etc. By using an adequate WT model the electrical power can be calculated. Once the power is known, the current through the IGBTs can be calculated. By using the actual temperature and current, the losses in the IGBTs can be calculated. By feeding the calculated losses in a thermal model, the junction temperature of the device (or the temperature at other layers of interest) can be obtained which can be used as an input for the lifetime prediction models.

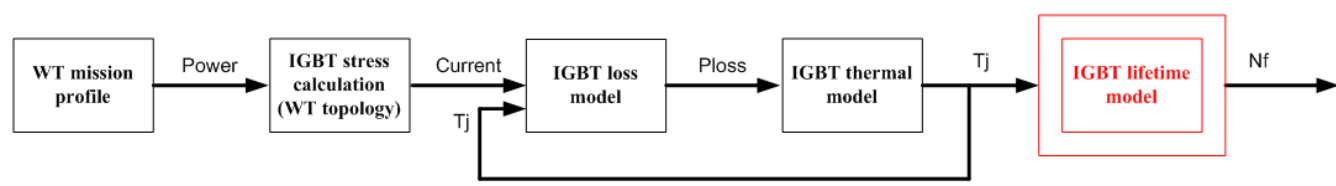

Fig. 1. IGBT lifetime prediction in WT applications.

According to [30] rotor side power converters in Doubly Fed Induction Generator (DFIG) WTs are stressed in a specific way due to the low output frequency. When a typical DFIG with two pole pairs is fed with $50 \mathrm{~Hz}$ it has a synchronous speed of $1500 \mathrm{rpm}$. Therefore the rotor side power converter has to operate typically in the frequency range of 0 to $16.6 \mathrm{~Hz}$ (depending on the slip of the DFIG). Therefore it has been chosen to simulate a classical two-level rotor side power converter with press-pack IGBTs in order to gain a better understanding of what is happening with the junction temperature of the devices in this kind of applications. The press-pack IGBT used for the simulation is the T1800GB45A made by Westcode. The simulated output current is $1200 \mathrm{~A}$, the frequency is $10 \mathrm{~Hz}$ and the switching frequency is $1 \mathrm{kHz}$. The simulated currents through the two IGBTs of one converter leg are shown in Fig. 2. The upper IGBT is conducting throughout the positive half cycle of the output current while the lower IGBT is conducting throughout the negative half 
cycle.

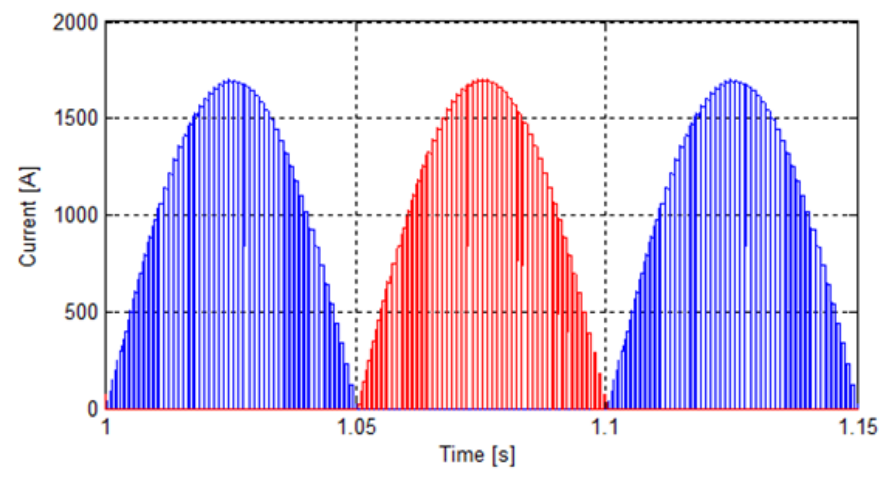

Fig. 2. The currents through the IGBTs of one converter leg.

The simulated junction temperature of one of the press-pack IGBTs is shown in Fig. 3. As it may be seen in this particular case the junction temperature swing is not large being only about $18{ }^{\circ} \mathrm{C}$ (from $101^{\circ} \mathrm{C}$ to $119{ }^{\circ} \mathrm{C}$ ).

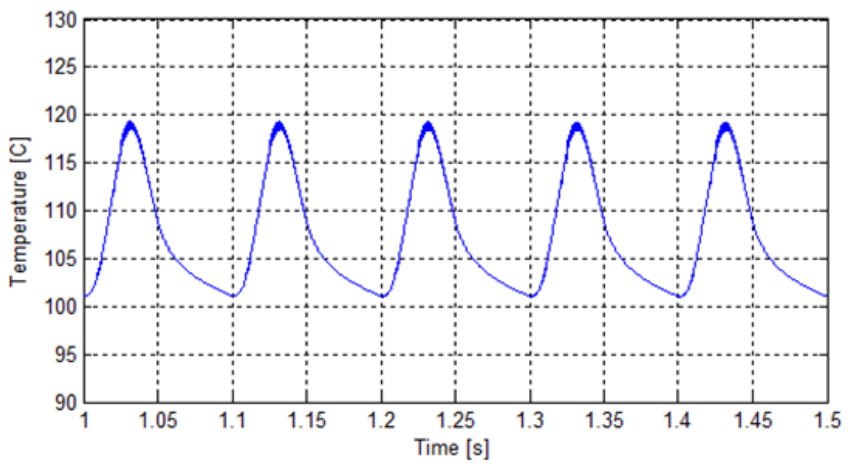

Fig. 3. The junction temperature of the upper IGBT.

\section{Conclusion}

Nowadays the reliability of power electronics is an important topic for researchers. Reliability is especially important for offshore wind turbines as the size and the number of installed WTs increases continuously. The repair cost and the value of the lost production in the case of failures can be high. The main objective of this paper was to review the reliability prediction related aspects of high power IGBTs in the context of wind power applications.

The dominant failure mechanisms of wire-bonded and press-pack IGBTs have been reviewed and compared. The most commonly used IGBT lifetime prediction models have been reviewed. Also the analytical models used to predict the life time of wire-bonded IGBT modules have been briefly compared. Press-pack IGBTs eliminate the dominant failure mechanisms of wire-bonded IGBT modules and offer better power cycling capability but at the same time introduce new failure mechanisms characteristic to press-pack devices. So far most of the reliability research was focused on the standard wire-bonded IGBT modules. The main reliability issue with both the wire-bonded and press-pack IGBTs is of thermomechanical nature (CTE mismatch combined with temperature variation).

More research is needed to be done about the failure mechanisms of press-pack IGBTs like the fretting damage, spring stress relaxation, spring fatigue, etc. Also new lifetime models for these new failure mechanisms characteristic to press-pack IGBTs need to be developed in order to allow WT manufacturers to design power converters with press-pack IGBTs for a certain reliability.

\section{Acknowledgements}

This work represents a part of the research carried out under the Vestas Power Programme at the Department of Energy Technology, Aalborg University, Denmark. The Vestas Power Programme is a collaboration programme between Vestas Wind Systems A/S and Aalborg University.

\section{References}

[1] European Wind Energy Association (EWEA), Pure Power - Wind energy targets for 2020 and 2030 (2009 update), December 2010, www.ewea.org. 
[2] European Wind Energy Association (EWEA), Wind energy factsheets, December 2010, www.ewea.org.

[3] S. Faulstich, B. Hahn, P. Lyding, P. Tavner, Reliability of offshore turbines - identifying risks by onshore experience, European offshore wind 2009, Conference and Exhibition, 14-16 Sept 2009, Stockholm, Sweden.

[4] J. Ribrant, L. M. Bertling, Survey of failures in wind power systems with focus on Swedish wind power plants during 1997-2005, IEEE Transactions on Energy Conversion, vol. 22, no. 1, pp.167-173, March 2007, DOI: 10.1109/TEC.2006.889614

[5] P. J. Tavner, J. Xiang, F. Spinato, Reliability analysis for wind turbines, Wind Energy 2007, 10:1-18, Wiley Interscience, DOI: $10.1002 /$ we.204.

[6] J. Lutz, H. Schlangenotto, U. Scheuermann, R. D. Doncker, Semiconductor Power Devices Physics, Characteristics, Reliability, First Edition, Springer, 2011, DOI: 10.1007/978-3-642-11125-9.

[7] I. F. Kovacevic, U. Drofenik, J. W. Kolar, New physical model for lifetime estimation of power modules, Power Electronics Conference (IPEC), 2010 International, pp. 2106 - 2114, DOI: 10.1109/IPEC.2010.5543755.

[8] M. Ciappa, Selected failure mechanisms of modern power modules, Microelectronics Reliability 42, pp. 653-667, 2002.

[9] N. D. Benavides, T. J. McCoy, M. A. Chrin, Reliability improvements in integrated power systems with pressure-contact semiconductors, December 2010, www.navalengineers.org.

[10] Westcode Semiconductors, December 2010, www.westcode.com.

[11] Toshiba Semiconductors, December 2010, www.semicon.toshiba.co.jp.

[12] Poseico, December 2010, www.poseico.com.

[13] A. Golland, F. Wakeman, G. Li, New family of 4.5kV press-pack IGBTs, Conference proceedings, June 2005, PCIM, Nuremberg, Germany.

[14] A. Pirondi, G. Nicoletto, P. Cova, M. Pasqualetti, M. Portesine, Thermo-mechanical finite element analysis in presspacked IGBT design, Microelectronics Reliability, 40, pp. 1163-1172, 2000.

[15] F. Wakeman, K. Billett, R. Irons, M. Evans, Electromechanical characteristics of a bondless pressure contact IGBT, Applied Power Electronics Conference and Exposition, 1999. APEC '99. Fourteenth Annual, pp. 312, DOI:

10.1109/APEC.1999.749659.

[16] P. Cova, G. Nicoletto, A. Pirondi, M. Portesine, M. Pasqualetti, Power cycling on press-pack IGBTs: measurements and thermo-mechanical simulation, Microelectronics Reliability, 39, 1165-1170, 1999.

[17] Z. Topolosky, Reliability analysis of springs used as interconnects in press-pack power electronic modules, University of Maryland, College Park, Master thesis, 2002.

[18] N. Kaminski, A. Kopta, Failure rates of HiPak modules due to cosmic rays, ABB Switzerland, July, 2009, www.abb.com.

[19] D. Chamund, D. Newcombe, IGBT module reliability, Application note, 2010, www.dynexsemi.com.

[20] M. Held, P. Jakob, G. Nicoletti, P. Scacco, M. H. Poech, Fast power cycling test of IGBT modules in traction application, Power Electronics and Drive Systems, 1997. Proceedings., 1997 International Conference on, pp 425, DOI: 10.1109/PEDS.1997.618742.

[21] M. Ciappa, F. Carbognani, P. Cova, W. Fichtner, Lifetime prediction and design of reliability tests for high-power devices in automotive applications, Reliability Physics Symposium Proceedings, 2003. $41^{\text {st }}$ Annual. 2003 IEEE International, pp. 523-528, DOI: 10.1109/RELPHY.2003.1197803.

[22] D. Hirschmann, D. Tissen, S. Schroder, R. W. D. Doncker, Reliability prediction for inverters in hybrid electrical vehicles, Power Electronics Specialists Conference, 2006. PESC '06. $37^{\text {th }}$ IEEE, pp. 1-6, DOI: 10.1109/PESC.2006.1712078.

[23] H. Cui, Accelerated temperature cycle test and Coffin-Manson model for electronic packaging, Reliability and Maintainability Symposium, 2005. Proceedings. Annual, pp. 556-560, DOI: 10.1109/RAMS.2005.1408421.

[24] R. Bayerer,T. Hermann, T. Licht, J. Lutz, M. Feller, Model for power cycling lifetime of IGBT modules - various factors influencing lifetime, in Proc. of the $5^{\text {th }}$ International Conference on Integrated Power Electronics Systems (CIPS), Nuremberg, Germany, March 11-13, 2008.

[25] M. Ciappa, F. Carbognani, W. Fichtner, Lifetime modelling of thermomechanics-related failure mechanisms in high power IGBT modules for traction applications, ISPSD, April 14-17, Cambridge, UK, 2003.

[26] P. Hansen, P. McCluskey, Failure models in power device interconnects, Power Electronics and Applications, 2007 European Conference on, pp. 1-9, DOI: 10.1109/EPE.2007.4417406.

[27] P. James, A. Forsyth, Accelerated testing of IGBT power modules to determine time to failure, Power Electronics, Machines and Drives (PEMD 2010), 5th IET International Conference on, pp. 1-4, DOI: 10.1049/cp.2010.0123.

[28] Reliasoft, Accelerated life testing reference, 2007, December 2010, www.weibull.com.

[29] M. Held, P. Jakob, G. Nicoletti, P. Scacco, M. H. Poech, Fast power cycling test of IGBT modules in traction application, Power Electronics and Drive Systems, 1997. Proceedings., 1997 International Conference on, pp. 425-430, vol.1, DOI: 10.1109/PEDS.1997.618742.

[30] M. Bartram, J. von Bloh, Rik W. De Doncker, Doubly-fed-machines in wind-turbine systems: Is this application limiting the lifetime of IGBT-frequency-converter?, Power Electronics Specialists Conference, 2004. PESC 04. 2004 IEEE 35th Annual, pp. 2583-2587, vol.4, DOI: 10.1109/PESC.2004.1355237. 